\title{
More than meets the eye in correcting refractive error in low income countries
}

\author{
Andrew Bastawrous clinical research fellow in international eye health ${ }^{1}$, Mutaz Aldawoud senior \\ clinician and clinical lead for service innovation ${ }^{2}$
}

${ }^{1}$ International Centre for Eye Health/Clinical Research Department, London School of Hygiene and Tropical Medicine, London WC1E 7HT, UK; ${ }^{2}$ Local Care Direct, Huddersfield, UK

Self correction of refractive error with adjustable spectacles is a diagnostic and treatment solution that could overcome the large unmet need for correcting refractive error, which is mostly caused by the lack of available trained refractionists and of suitable, available, and affordable spectacles for those in need. ${ }^{1}$ Acceptance of spectacle wear is often poor, even when spectacles are provided free of charge and greatly improve vision. Reasons include cosmesis, fear of teasing by peers, the notion that spectacles identify people as having a disability, and the belief that vision worsens with continued spectacle wear. ${ }^{23}$ To improve long term adherence, these innovative spectacles need to be made fashionably and sociably acceptable. A variety of frame designs and colours, together with local celebrities or respected figures using them in public, are likely to increase uptake and adherence.

Competing interests: None declared.

1 Zhang M, Zhang R, He M, Liang W, Li X, She L, et al. Self correction of refractive error among young people in rural China: results of cross sectional investigation. $B M J$ 2011;343:d4767. (9 August.)

2 Castanon Holguin AM, Congdon N, Patel N, Ratcliffe A, Esteso P, Toledo Flores S. Factors associated with spectacle-wear compliance in school-aged Mexican children. Invest Ophthalmol Vis Sci 2006;47:925-8.

3 Adeoti CO. Beliefs and attitude towards spectacles. Niger J Clin Pract 2009;12:359-61.

๑ BMJ Publishing Group Ltd 2011 